\title{
Numerical Investigation of Flow in an Over-expanded Nozzle with Porous Surfaces
}

\author{
Alaa Elmiligui* \\ Analytical Services \& Materials, Inc. Hampton, VA 23666 \\ K.S. Abdol-Hamid ${ }^{\dagger}$ and Craig A. Hunter ${ }^{*}$ \\ NASA Langley Research Center, Hampton VA 23681
}

\begin{abstract}
A new porous condition has been implemented in the PAB3D solver for simulating the flow over porous surfaces. The newly-added boundary condition is utilized to compute the flow field of a non-axisymmetric, convergent-divergent nozzle incorporating porous cavities for shock-boundary layer interaction control. The nozzle has an expansion ratio (exit area/throat area) of $\mathbf{1 . 7 9 7}$ and a design nozzle pressure ratio of 8.78 . The flow fields for a baseline nozzle (no porosity) and for a nozzle with porous surfaces $(10 \%$ porosity ratio) are computed for NPR varying from 2.01 to 9.54. Computational model results indicate that the over-expanded nozzle flow was dominated by shock-induced boundary-layer separation. Porous configurations were capable of controlling off-design separation in the nozzle by encouraging stable separation of the exhaust flow. Computational simulation results, wall centerline pressure, mach contours, and thrust efficiency ratio are presented and discussed. Computed results are in excellent agreement with experimental data.
\end{abstract}

\section{Introduction}

$\mathrm{I}_{\mathrm{i}}^{\mathrm{n}}$ nvestigations in the area of Passive Porosity Technology (1-9) for propulsion applications have led to an increased interest in upgrading PAB3D's (10) modeling capabilities. PAB3D is a structured, multi-block, parallel, implicit, finite-volume solver of the three-dimensional, unsteady, Reynolds-Averaged Navier-Stokes equations. Advanced turbulence models are available in PAB3D and are widely used in internal and external flow applications by NASA and the U.S. aerospace industry. In PAB3D, second-order time accuracy can be achieved by employing physical time sub-iteration and dual time sub-iteration (13). In an attempt to increase the fidelity and flexibility of PAB3D, a new boundary condition for simulation of the flow over porous surfaces has been added. Porous boundary simulates a porous surface placed above a plenum. The model eliminates the need for construction of a grid within an underlying plenum, thereby simplifying the numerical modeling of passive porous control systems $(8,9)$.

Hunter $(1,3)$ performed an experimental investigation on a non-axisymmetric, convergent-divergent nozzle incorporating porous cavities for shock-boundary layer interaction control. The experimental testing on the nozzle was performed in the 16-Foot Transonic Tunnel Complex at NASA Langley Research Center (LaRC) as part of a comprehensive static performance investigation. Force, moment, and pressure measurements were made and schlieren flow visualization was obtained for a subscale, non-axisymmetric, two-dimensional, convergent-divergent nozzle. Details of the nozzle are shown in Figure 1, and a photograph of the nozzle is shown in Figure 2. Results by Hunter (5) indicate that over-expanded nozzle flow was dominated by shock-induced boundary-layer separation, which was divided into two distinct flow regimes: three-dimensional separation with partial reattachment, and fully detached two-dimensional separation. For nozzle pressure ratio, NPR $\leq 1.8$, the separation was three-dimensional, somewhat unsteady, and confined to a bubble (with partial reattachment over the nozzle flap). For NPR $\geq 2.0$, separation was steady and fully detached, and it became more two-dimensional as NPR increased. When NPR increased from 1.8 to 2.0, the nozzle went through a dramatic transition, dividing the two separated flow regimes. Figure 3 shows schematic of a flow in a shock-separated nozzle.

With the implementation of the new porous boundary condition in PAB3D, a detailed numerical investigation of the nozzle can be conducted, and numerical results can be compared to experimental data. In the present study, a

\footnotetext{
${ }^{*}$ Senior Research Scientist, AIAA Member.

${ }^{\dagger}$ Aerospace Engineer, Configuration Aerodynamics Branch, MS 499, Associate Fellow AIAA.

*Aerospace Engineer, Configuration Aerodynamics Branch, MS 499.
} 
two-dimensional computational model is used to compute the flow of the over-expanded nozzle for NPR $>2.0$. In a parallel effort, a three-dimensional model is being constructed to compute flow for NPR $\leq 2.0$. This study is a continuation of the work of Hunter $(4,5)$, and will serve as the validation case for the implementation of the porous surface boundary condition.

The objective of this study is to implement, validate and examine the newly added porous boundary condition of PAB3D and computationally determine the internal performance (nozzle thrust ratio) of a fixed geometry exhaust nozzle incorporating porous cavities for shock boundary layer interaction control for NPR $>2.0$.

The organization of this paper will be as follows: The governing equations and boundary conditions are presented followed by a detailed computational study of a convergent-divergent nozzle incorporating porous cavities. Numerical results for both a baseline configuration and porous configurations are presented, discussed and compared to experimental data (3). Physical phenomena of passive porous control are highlighted, and flow characteristics are described.

\section{Computational Fluid Dynamics Simulation}

In this study, PAB3D is used in conjunction with two-equation k- $\varepsilon$ turbulence closure and nonlinear algebraic Reynolds stress models to simulate separated nozzle flows. PAB3D has been well-tested and documented for the simulation of aero-propulsive and aerodynamic flows involving separation, mixing, and other complicated phenomena (10-13). PAB3D is ported to a number of platforms and offers a combination of good performance and low memory requirements. In addition to its advanced preprocessor, which can handle complex geometries through multi-block general patching, PAB3D has a runtime module capable of calculating aerodynamic performance on the fly and a postprocessor used for follow-on data analysis (14). PAB3D solves the simplified Reynolds-averaged Navier-Stokes equations in conservative form, obtained by neglecting stream-wise derivatives of the viscous terms. Viscous models include coupled and uncoupled simplified Navier-Stokes and thin layer Navier-Stokes options. Roe`s upwind scheme is used to evaluate the explicit part of the governing equations, and van Leer's scheme is used for the implicit part. Diffusion terms are centrally differenced, inviscid terms are upwind differenced, and two finite volume flux-splitting schemes are used to construct the convective flux terms. PAB3D is third-order accurate in space and second-order accurate in time.

\section{Governing Equations}

The governing equations solved in this study are the time-averaged Reynolds Averaged Navier-Stokes (RANS), and the perfect gas law is chosen to represent the air properties. The mass, momentum, and energy conservation equations can be written in a conservative form as follows:

$$
\begin{aligned}
& \frac{\partial \rho}{\partial t}+\frac{\partial \rho u_{i}}{\partial x_{i}}=0 \\
& \frac{\partial \rho u_{i}}{\partial t}+\frac{\partial\left(\rho u_{i} u_{j}+p \delta_{i j}\right)}{\partial x_{j}}=\frac{\partial\left(\tau_{i j}-\rho \overline{u_{i} u_{j}}\right)}{\partial x_{j}} \\
& \frac{\partial \rho e_{0}}{\partial t}+\frac{\partial\left(\rho e_{0} u_{i}+p u_{i}\right)}{\partial x_{i}}=\frac{\partial\left(\tau_{i j} u_{j}-\rho \overline{u_{i} u_{j}} u_{j}\right)}{\partial x_{i}}-\frac{\partial\left(q_{i}+C_{p} \rho \overline{u_{i} \theta}\right)}{\partial x_{i}}+\frac{\partial}{\partial x_{i}}\left[\left(\mu_{l}+\frac{\mu_{t}}{\bar{\sigma}_{k}}\right) \frac{\partial k}{\partial x_{i}}\right]
\end{aligned}
$$

Three turbulence models are used in the current study to model turbulence: a standard k- $\varepsilon$ model (11) and two algebraic stress models: Shih-Zhu-Lumley (SZL) (16), and Girimaji model (17). The two algebraic stress models give inherently better results than the linear stress model because of the explicit modeling of effects such as relaxation, and the specific inclusion of nonlinear anisotropic effects from the mean flow strain and vorticity. With a nonlinear model, the calculation of six independent, realizable Reynolds stress terms is possible. This type of detail is important for simulating complicated multidimensional flows. In the two-equation (k-€ turbulence model), the eddy-viscosity concept is used to model the Reynolds stresses, and equations are: 


$$
\begin{aligned}
& \frac{\partial \rho k}{\partial t}+\frac{\partial \rho u_{j} k}{\partial x_{j}}=-\rho \bar{u}_{j} u_{i} \frac{\partial u_{i}}{\partial x_{j}}+\frac{\partial}{\partial x_{j}}\left[\left(\mu_{l}+\frac{c_{\mu} \rho k^{2}}{\bar{\sigma}_{k} \varepsilon}\right) \frac{\partial k}{\partial x_{j}}\right]-\rho \varepsilon\left(1 .+M_{\tau}^{2}\right) \\
& \frac{\partial \rho \varepsilon}{\partial t}+\frac{\partial \rho u_{j} \varepsilon}{\partial x_{j}}=-C_{\varepsilon 1} \rho \overline{u_{j} u_{i}} \frac{\partial u_{i}}{\partial x_{j}} \frac{\varepsilon}{k}+\frac{\partial}{\partial x_{j}}\left[\left(\mu_{l}+\frac{c_{\mu} \rho k^{2}}{\bar{\sigma}_{\varepsilon} \varepsilon}\right) \frac{\partial \varepsilon}{\partial x_{j}}\right]-f_{2} \widetilde{C}_{\varepsilon 2} \rho \frac{\varepsilon}{k}\left[\varepsilon-v_{l}\left(\frac{\partial \sqrt{k}}{\partial h}\right)^{2}\right] \\
& f_{\mu}=\exp \left[\frac{-3.41}{\left(1+\frac{R_{T}}{50}\right)^{2}}\right], R_{T}=\frac{k^{2}}{\mu_{t} \varepsilon}, f_{2}=1 .-0.3 \exp \left(-R_{T}^{2}\right)
\end{aligned}
$$

The boundary conditions for $\varepsilon$ and $\mathrm{k}$ at the wall are:

$$
\begin{gathered}
\varepsilon_{\text {wall }}=v_{l}\left(\frac{\partial \sqrt{k}}{\partial n}\right)^{2} \\
k_{\text {wall }}=0
\end{gathered}
$$

The turbulent stress components are formulated as:

$$
\begin{aligned}
& \rho \overline{u_{j} u_{i}}=2 \rho v_{t} S_{j i}-\frac{2}{3} \delta_{j i} \rho k \\
& S_{j i}=\frac{1}{2}\left\lfloor\frac{\partial u_{j}}{\partial x_{i}}+\frac{\partial u_{i}}{\partial x_{j}}\right\rfloor-\frac{1}{3} \delta_{j i} \frac{\partial u_{j}}{\partial x_{i}} \\
& v_{t}=f_{\mu} C_{\mu}^{*} \frac{k^{2}}{\varepsilon}
\end{aligned}
$$

$C_{\mu}^{*}$ is 0.09 for the linear model and is a function of vorticity and strain tensors for the nonlinear models. In the SZL nonlinear model (16), the turbulent stresses are given by:

$-\overline{u_{j} u_{i}}=-\frac{2}{3} \delta_{j i} k+2 v_{t} S_{j i}-2 \beta \frac{K^{3}}{\varepsilon^{2}}\left(W_{i k} \bar{S}_{k j}-\bar{S}_{i k} W_{k j}\right)$

Stresses in the Girimaji nonlinear model (17) are given by: 
$-\overline{u_{j} u_{i}}=-\frac{2}{3} \delta_{j i} k+2 v_{t} S_{j i}-2 C_{\mu}^{*} \frac{K^{3}}{\varepsilon^{2}}\left[-G_{2}\left(W_{i k} S_{k j}-S_{i k} W_{k j}\right)+G_{3}\left(S_{i k} S_{k j}-\frac{1}{3} S_{m n} S_{m n} \delta_{i j}\right)\right]$

where

$W_{i j}=\frac{1}{2}\left(\frac{\partial u_{i}}{\partial x_{j}}-\frac{\partial u_{j}}{\partial x_{i}}\right)$

$\bar{S}_{i j}=S_{i j}-\frac{1}{3} S_{k k} \delta_{i j}$

A compilation of the parameters used in the turbulence models can be found in the Appendix.

\section{Computational Domain}

The computational grid used in this study is shown in Figure 4. The two-dimensional grid consisted of 66,400 cells and 5 blocks. Relative to the nozzle exit, the grid extended approximately 30 throat heights downstream, 25 throat heights upstream, and 25 throat heights normal to the jet axis. In an attempt to capture the complicated physics of the shock-boundary layer interaction process, the divergent section of the nozzle was densely gridded with cells having an aspect ratio near 1:1. There were approximately 40 cells in the boundary layer grid with first cell height of approximately $y+=0.5$. An inflow duct (sized like the instrumentation duct used in the experimental study) was located upstream of the nozzle.

\section{Initial and Boundary Conditions}

Stagnation conditions were applied to the left face of the inflow duct upstream of the nozzle, and were chosen to match experimental conditions for total pressure and temperature (3). In addition, an initial Mach number was specified in the inflow block and nozzle to start the solution. The static ambient region surrounding the nozzle was defined by a subsonic inflow condition $\left(\mathrm{T}_{\mathrm{a}}=530^{\circ} \mathrm{R}, \mathrm{P}_{\mathrm{a}}=14.85 \mathrm{psi}, \mathrm{M}_{\mathrm{a}}=0.025\right)$ on the left face, a characteristic boundary condition on the top face, and a smart boundary condition on the right face that switched between constant pressure outflow (subsonic) and first order extrapolation (supersonic), depending on the local Mach number. All solid walls were treated as no-slip adiabatic surfaces, and the bottom of the entire domain was defined by a symmetry boundary condition. A porous boundary condition was specified for the porous surface and porous cavity. Porous boundary simulates a porous surface placed above a plenum. The model eliminates the need for construction of a grid within an underlying plenum, thereby simplifying the numerical modeling of passively porous flow control systems, and reduces computation cost. A sketch showing the porous cavity concept for shockboundary layer interaction control is shown in Figure 5. The Porous patch allows the high-pressure region behind the shock to communicate with the low-pressure region ahead of the shock, which reduces shock strength and consequently reduces adverse effects of shock on the boundary layer separation. The proposed porous surface boundary implemented into PAB3D builds on the work of Ref. $(8,9)$. In the present implementation of the porous boundary condition, plenum pressure that yields a zero net mass flux balance across porous patch is computed. The normal velocity is used to determine the flow direction rather than the plenum pressure as suggested in Ref (9). This modification to the original porous boundary was found to enhance convergence.

In the experimental study of Ref (3), one baseline (no porosity) and 27 porous configurations were tested. The variation in the porosity percentage $(10 \%, 20 \%$, and $30 \%)$, hole diameter $d(0.025$ in., 0.052 in., and $0.076 \mathrm{in}$.), and cavity depth D (shallow, medium, and deep) resulted in 27 nozzle configurations. One limitation of the current porous boundary condition is the fact that it models only porosity ratio and is insensitive to the hole diameter and the cavity depth. Thus the present porous boundary condition simulates experimental nozzle configurations with the smallest hole diameter and cavity depth.

\section{Results}

An assessment of the new porous boundary condition was made by solving the flow in a non-axisymmetric, convergent-divergent nozzle incorporating porous cavities for shock-boundary layer interaction control. Computational simulations were performed at nozzle pressure ratios from 2.01 to 9.5 for a baseline configuration (no porosity) and for a nozzle with $10 \%$ porosity ratio. These conditions were chosen for detailed comparison with experimental data (3). Numerical results are presented in terms of internal flow features (static pressure, thrust performance, mach contours and schlieren flow visualization). Grid sequencing is used to accelerate convergence by 
solving $1 / 4$ then $1 / 2$ of the grid in each of the three computational directions. Each of the 2D simulations performed in this study ran 5,000 iterations at each grid level.

Prior to conducting the detailed computational analysis of the non-axisymmetric nozzle flow, test cases were run for NPR=3.0 in order to assess PAB3D performance. This includes monitoring net mass flow through the porous patch, grid convergence and the performance of various turbulent models, using experimental centerline pressure data as the criterion. At this NPR, experimental results showed shock-boundary layer interaction, and pressure data indicated that nozzle flow was nearly 2D. Figure 6 shows the net mass flux across a porous patch approached zero in less than 1000 iterations. Figure 7 shows the convergence history for the baseline configuration at NPR=3.0 while Figure 8 shows the computed wall static pressure $\left(\mathrm{P} / \mathrm{P}_{\mathrm{oj}}\right)$ for all grid levels, versus non-dimensional stream-wise location. The coarse grid level failed to capture the shock accurately while medium and fine grid levels were in excellent agreement with experimental data of Ref. (3). This demonstrates numerical verification for PAB3D with respect to grid distribution.

Figure 9 shows a comparison of computational and wall centerline experimental pressures over the entire nozzle length at NPR $=3.0$ for all three turbulence models used in this study. All three models showed excellent agreement with experimental pressures upstream and immediately downstream of the nozzle throat. Further downstream, all three models appeared to model the shock-boundary layer interaction well with SZL giving the best comparison with experimental data (3). CFD results shown hereafter will be for SZL model.

\section{Baseline Configuration (No Porosity)}

Figure 10 shows comparison between the computed and experimental wall centerline static pressures $\left(\mathrm{P} / \mathrm{P}_{\mathrm{oj}}\right)$, plotted against non-dimensional stream-wise location for the baseline nozzle configuration at off-design nozzle conditions for NPR $\leq 5.423$. CFD results are shown as the solid lines, while symbols represent experimental data of Ref. (3). Experimental and computational Schlieren flow visualization are shown in Figure 11 while Mach Contours for nozzle flow are presented in Figure 12. Results are representative of classic convergent-divergent nozzle flow. Schlieren flow visualization at NPR $=2.0$ shows the nozzle shock with a pronounced lambda foot system and fully detached separation extending from the leading lambda shock downstream past the nozzle exit. Fully detached separation occurred for subsequent NPRs past 2.0. Increasing NPR forced lambda shock to increase in size and move downstream. By NPR=3.4, the lambda shock foot had grown significantly, such that the main shock and trailing lambda foot were outside the physical nozzle, as shown in Figure 11. At this NPR, flow past the separation point showed strong resemblance to externally over-expanded flow; the jet plume necked down between the leading and trailing lambda foot, and there was an expansion fan emanating from each trailing lambda foot as it intersected the free shear layer. Figure 11 shows a good qualitative agreement between computational and experimental schlieren images, though the computational simulation is seen to predict more of a "stretched" shock structure. With increasing NPR, the leading lambda foot worked its way out of the nozzle, and pressure data and flow visualization shows the nozzle to be internally shock-free. Figure 13 shows excellent agreement between computational and experimental wall centerline pressure data for NPR $\geq 5.4$ and shows the nozzle to be shock-free and all pressures situated on the same curve.

The internal flow discrepancies noted between computed results and experimental data at NPR=2.0 are due to the 2D nature of the computational simulation, and the fact that the flow has not yet recovered from the threedimensional flow region. The fact that the flow began to take on a $2 \mathrm{D}$ nature at around NPR=2.21, where computation and experiments begin to agree, indicates that a 3D simulation is necessary to correctly model nozzle flow at low NPRs.

\section{Porous Configurations}

Computational simulations were performed at nozzle pressure ratios from 2.01 to 9.5 . Figure 14 shows comparison between the computed and experimental wall centerline static pressures (P/Poj), plotted against nondimensional stream-wise location for a nozzle configuration with a $10 \%$ porosity patch at off-design nozzle conditions for $2.0<\mathrm{NPR} \leq 5.423$. Computed results agree well with experimental data (3). The porous patch affected static pressure ratio distributions in the nozzle across the entire operating range compared to baseline configuration. The nozzle shock is located over the porous region for NPRs between 2.01 and 4.21. Figure 15 shows mach contours, for NPR $=2.41$, for nozzle flow with and without porosity at NPR=2.41. Comparison of computed wall pressure distribution for baseline configuration and a 10\% porous patch configuration, for NPR=2.4 and 3.01, are shown in are shown in Figure 16. Computed schlieren images for the 10\% porosity configuration are shown in Figure 17. At NPR=3.41 where the shock location had moved to the very downstream end of the porous patch, porosity provided little apparent separation control and the only significant difference in the static pressure ratio 
distributions between the porous configuration and the baseline configuration is a more gradual compression through the shock for the porous configuration.

\section{Performance}

Figure 18 shows the computed thrust ratio $\mathrm{F} / \mathrm{F}_{\mathrm{i}}$ plotted against NPR, accompanied by experimental data of (3), for the baseline configuration and the porous configuration. Peak Thrust ratio for porous configurations was lower than that of the baseline configuration. The computational simulation did a good job of modeling the overall thrust efficiency trend but failed to predict the on-design thrust efficiency. This is to be expected since the 2D computational model does not account for viscous effects on the nozzle sidewalls.

\section{Summary and Future Work}

A new porous boundary condition, for simulating the flow over porous surfaces, has been successfully added to PAB3D. The porous boundary condition reduces computation cost and simplifies the numerical modeling of passive porous surfaces. The porous boundary condition was utilized to compute the flow field of a non-axisymmetric, convergent-divergent nozzle incorporating porous cavities for shock-boundary layer interaction control. Numerical results indicate that baseline (no porosity) nozzle performance is dominated by unstable, shock-induced, boundarylayer separation at over-expanded conditions, and that porous configurations were capable of controlling off-design separation in the nozzle by encouraging stable separation of the exhaust flow. Computational results are in excellent agreement with experimental data for NPRs greater than 2.0. Future work will involve building a 3D computational model to accurately model lower NPR and to study nozzle separation in general.

\section{Appendix Algebraic Stress Models Equation Parameters}

The following functions and variables were used in the algebraic Reynolds stress models used by the SZL (16) model:

$$
\begin{gathered}
C_{\mu}^{*}=\left(\begin{array}{c}
1 \\
\left.6.5+A_{s}^{*} \frac{U^{*} k}{\varepsilon}\right)
\end{array}\right. \\
C_{\mu}^{*}=\frac{1}{\left(6.5+A_{s}^{*} \frac{U^{*} k}{\varepsilon}\right)} \quad A_{s}^{*}=\sqrt{6} \cos (\phi) \\
S^{*}=\sqrt{S_{i j}^{*} S_{i j}^{*}} \quad \Omega^{*}=\sqrt{\Omega_{i j}^{*} \Omega_{i j}^{*}} \cos ^{-1}\left(\sqrt{6} W^{*}\right. \\
S_{i j}^{*}=S_{i j}-\frac{1}{3} S_{k k} \delta_{i j} \quad \Omega_{i j}^{*}=\Omega_{i j}=\frac{1}{2}\left(\frac{\partial u_{i}}{\partial x_{j}}-\frac{\partial u_{j}}{\partial x_{i}}\right)=-\Omega_{i j} \\
W^{*}=S_{i j}^{*} S_{j k}^{*} S_{k i}^{*} /\left(S^{*}\right)^{3} \\
U^{*}=\sqrt{S_{i j}^{*} S_{i j}^{*}+\Omega_{i j}^{*} \Omega_{i j}^{*}} \quad \beta=\frac{\sqrt{1-9 C_{\mu}^{2}\left(\frac{S^{*} k}{\varepsilon}\right)^{2}}}{\left(1+6 \frac{S^{*} k}{\varepsilon} \frac{\Omega^{*} k}{\varepsilon}\right)}
\end{gathered}
$$

The following functions and variables were used in the algebraic Reynolds stress models used by Girimaji (17) model:

$$
\begin{array}{ll}
L_{1}^{0}=\frac{C_{1}^{1}}{2}+1 & L_{1}^{1}=C_{1}^{1}+2 \\
L_{2}=\frac{C_{2}}{2}-\frac{2}{3} & L_{3}=\frac{C_{2}}{2}-1 \quad L_{4}=\frac{C_{4}}{2}-1
\end{array}
$$




$$
\begin{gathered}
\eta_{1}=\left(\frac{k}{\varepsilon}\right)^{2} S_{m n} S_{m n} \quad p=-\frac{2 L_{1}^{0}}{\eta_{1} L_{1}^{1}} \\
\eta_{2}=\left(\frac{k}{\varepsilon}\right)^{2} W_{m n} W_{m n} \quad r=-\frac{L_{1}^{0} L_{2}}{\left(\eta_{1} L_{1}^{1}\right)^{2}} \\
\left.q=\frac{1}{\left(\eta_{1} L_{1}^{1}\right)^{2}} \mid\left(L_{1}^{0}\right)^{2}+\eta_{1} L_{1}^{1} L_{2}-\frac{2}{3} \eta_{1}\left(L_{3}\right)^{2}+2 \eta_{2}\left(L_{4}\right)^{2}\right] \\
a=\left(q-\frac{p^{2}}{3}\right) \quad b=\frac{1}{27}\left(2 p^{3}-9 p q+27 r\right) \\
D=\frac{b^{2}}{4}+\frac{a^{3}}{27} \quad \cos (\theta)=\frac{-b / 2}{\sqrt{-a^{3} / 27}}
\end{gathered}
$$

The coefficients $\mathrm{G}_{2}$ and $\mathrm{G}_{3}$ are

$$
\begin{aligned}
& G_{2}=\frac{-L_{4} G_{1}}{L_{0}^{1}-\eta_{1} L_{1}^{1} G_{1}} \quad G_{3}=\frac{-L_{3} G_{1}}{L_{0}^{1}-\eta_{1} L_{1}^{1} G_{1}}
\end{aligned}
$$

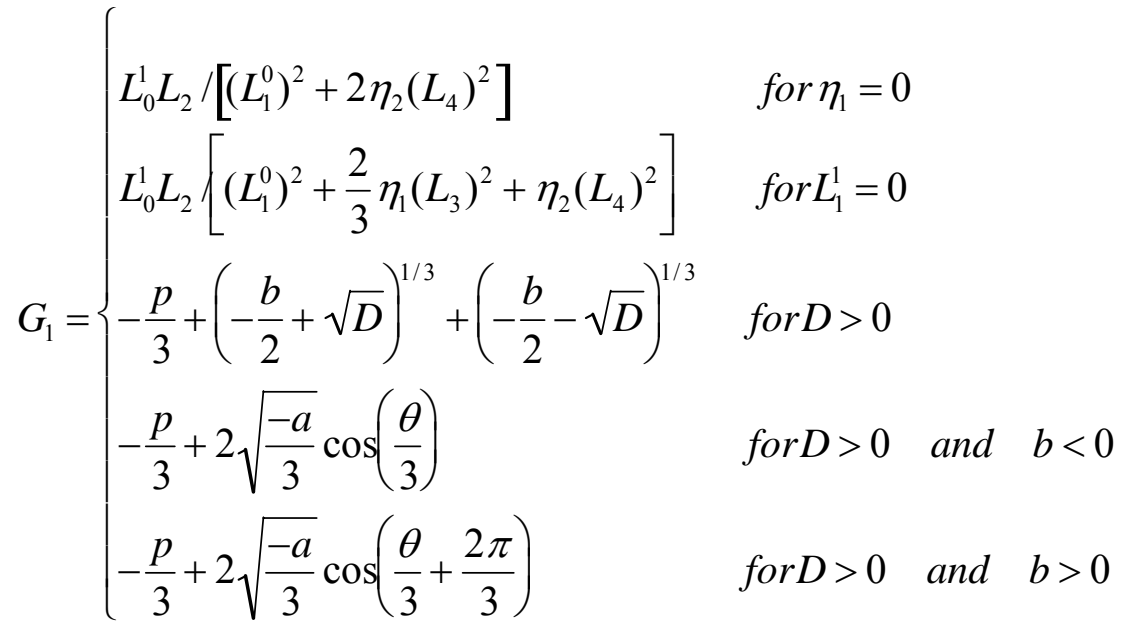

$$
\begin{aligned}
& C_{\mu}^{*}=-G_{1} \\
& C_{1}^{0}=3.4 \quad C_{1}^{1}=1.8 \quad C_{2}=0.36 \quad C_{3}=1.25 \quad C_{4}=0.4
\end{aligned}
$$

\section{Acknowledgments}

This work is part of a larger ongoing study of complex nozzle configurations. A jet noise prediction method based on computational information is also being pursued together with the computational results and the experiments. The authors would like to thank Dr. Russ Thomas and Dr. S. Paul Pao, from NASA Langley Research Center, and Dr. Steven Massey from Eagle Aeronautics, for long hours of fruitful discussions. The first author would like to acknowledge the support of NASA Langley Research Center for providing the funding needed to carry out this work.

\section{References}

1. Craig A. Hunter, Sally A. Viken, Richard M. Wood, and Steven X. S. Bauer. "Advanced Aerodynamic Design of Passive Porosity Control Effectors". AIAA Paper 2001-249, Jan. 2001

2. Scott C. Asbury, Christopher L. Gunther, and Craig A. Hunter. "A passive cavity concept for improving the offdesign performance of fixed-geometry exhausts nozzles." AIAA Paper 1996-2541

3. Scott C. Asbury and Craig A. Hunter. "Static performance of a fixed-Geometry Exhaust Nozzle Incorporating Porous Cavities for Shock-Boundary Layer Interaction". NASA TM-1999-209513 
4. Hunter, C.A. "Experimental, Theoretical, and Computational Investigation of Separated Nozzle Flows." AIAA Paper 1998-3107, 1998

5. Hunter, C.A. ""Experimental Investigation of Separated Nozzle Flows." Journal of Propulsion and Power. Volume 20, Number 3, May-June 2004, 527-532

6. J. Lopera, T. Terry, M. Patel. "Experimental Investigations of Reconfigurable Porosity for Aerodynamic Control." 2nd AIAA Flow Control Conference, Portland, Oregon, June 2004. AIAA-2004-2695.

7. M. Patel, J. DiCocco, T. Prince, T. Ng. "Flow Control Using Reconfigurable Porosity" 21st AIAA Applied Aerodynamics Conference, Orlando, Florida, June 23-26, 2003, AIAA-2003-3665.

8. Ana F. Tinetti. "On the Use of Surface Porosity to Reduce Wake-Stator Interaction Noise." Ph.D. Thesis, Virginia Polytechnic Institute and State University, September 2001.

9. Neal T. Frink; Daryl L. Bonhaus; Veer N. Vatsa; Steven X. S. Bauer; Ana F. Tinetti. "Boundary Condition for Simulation of Flow over Porous Surfaces" Journal of Aircraft, Vol. 40, Number 4, 2003, pp. 692-698.

10. PAB3D Code Manual Originally developed by the Propulsion Aerodynamics Branch, now under cooperative program between the Configuration Aerodynamics Branch, NASA Langley Research Center and Analytical Services \& Materials, Inc. Hampton, VA. See http://www.asm-usa.com/software/pab3d.html

11. Abdol-Hamid, K.S. "Implementation of Algebraic Stress Models in a General 3-D Navier-Stokes Method (PAB3D)". NASA CR-4702, December 1995.

12. Carlson, J. R. "Applications of Algebric Reynolds Stress Turbulence Models" Journal of Propulsion and Power. Vol. 13 No. 5. 1997

13. Massey, S.J., \& Abdol-Hamid, K.S, "Enhancement and Validation of PAB3D for Unsteady Aerodynamics," AIAA Paper 2003-1235, 2003.

14. Massey, S. J., POST Code Manual, See http://eagle.com/post/

15. Leavitt, L.D., and Re, R.J. "Static Internal Performance Including Thrust Vectoring and Reversing of TwoDimensional Convergent-Divergent Nozzles". NASATP-2253, 1984.

16. Shih, T.H., Zhu, J., and Lumley, J.L. "A New Reynolds Stress Algebraic Equation Model". NASA TM-106644, August 1994.

17. Girimaji, S.S. "Fully-Explicit and Self-Consistent Algebraic Reynolds Stress Model". ICASE 95-82, December 1995. 


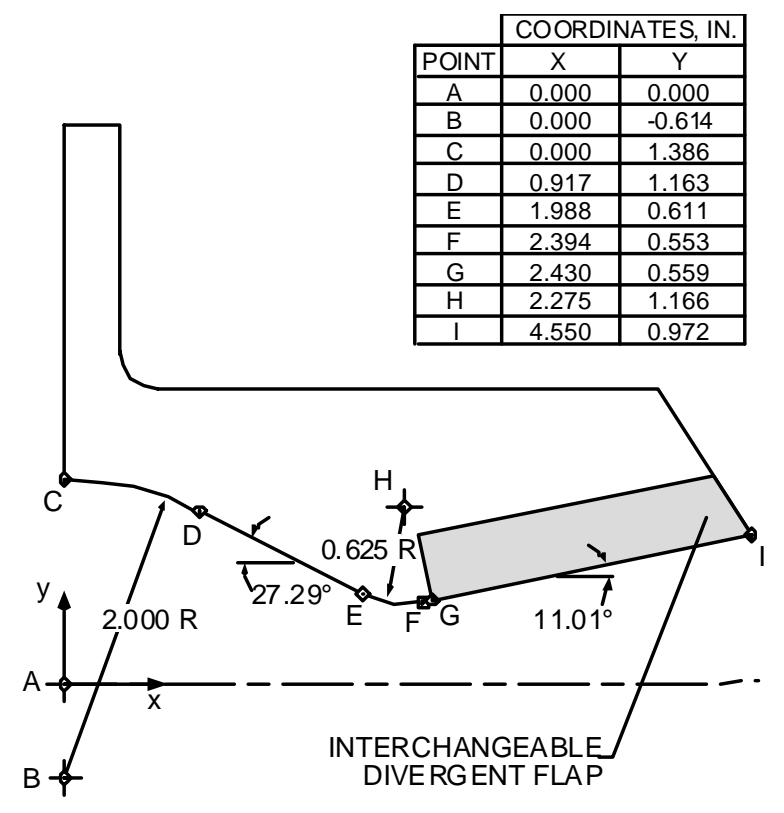

Figure 1. Sketch showing nozzle geometric details, Ref. (3)

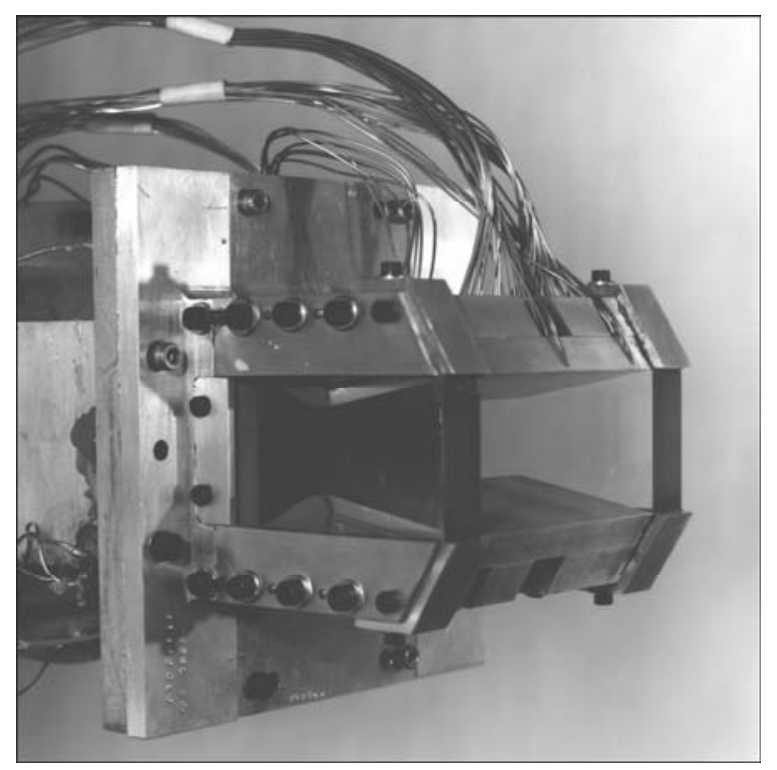

Figure 2. Non-axisymmetric convergent divergent nozzle, Ref. (5)

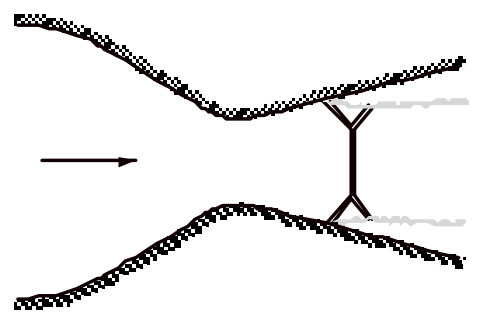

Figure 3. Schematic of over-expanded nozzle with separation. 


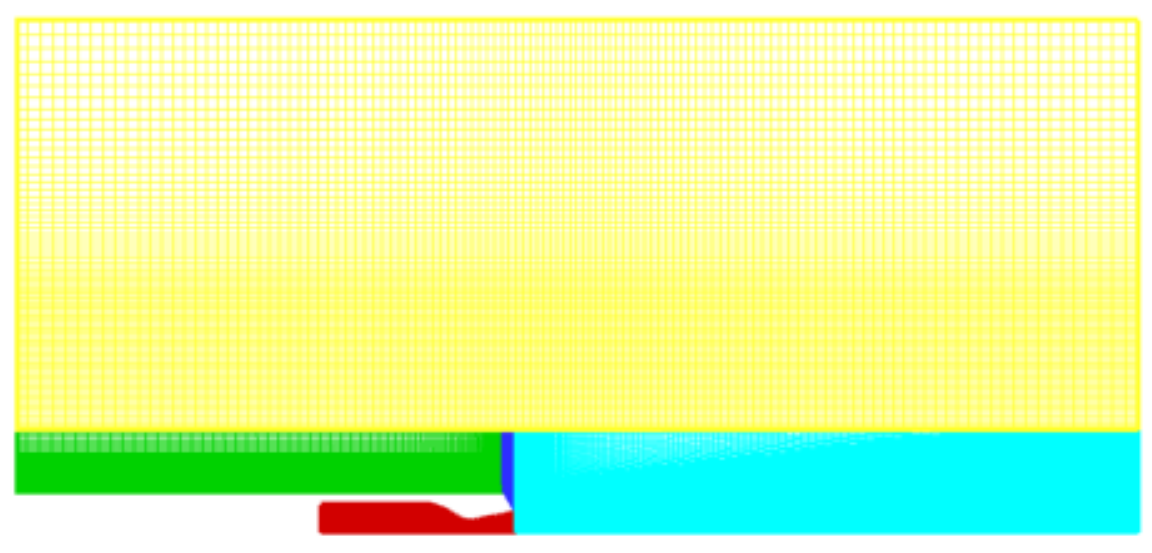

Figure 4. Computational grid

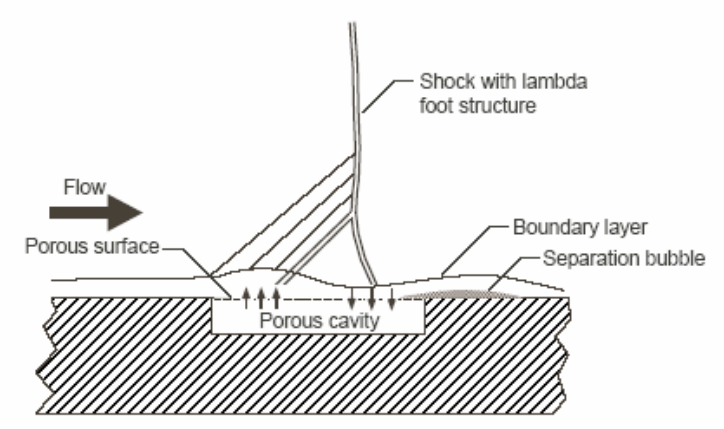

Figure 5. Sketch showing the porous cavity concept for shock-boundary layer interaction control, Ref (3)

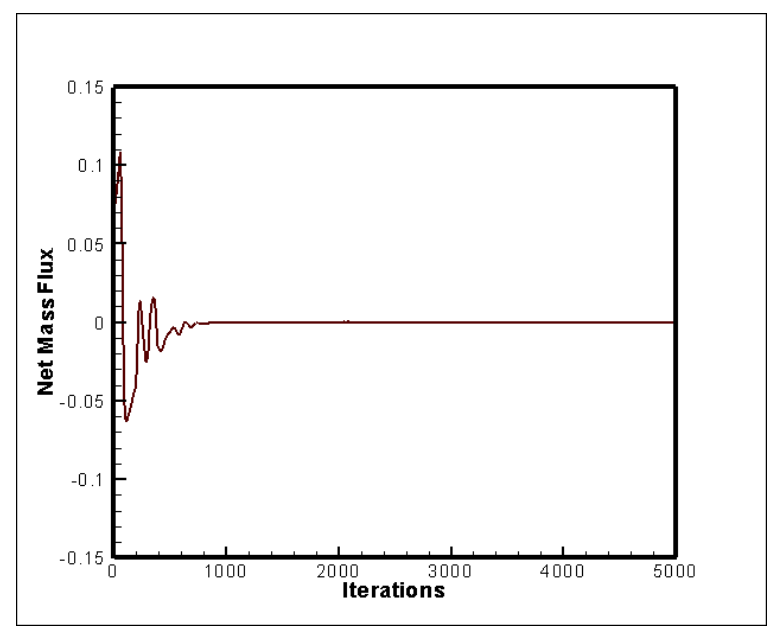

Figure 6. Net mass flux across porous patch. 


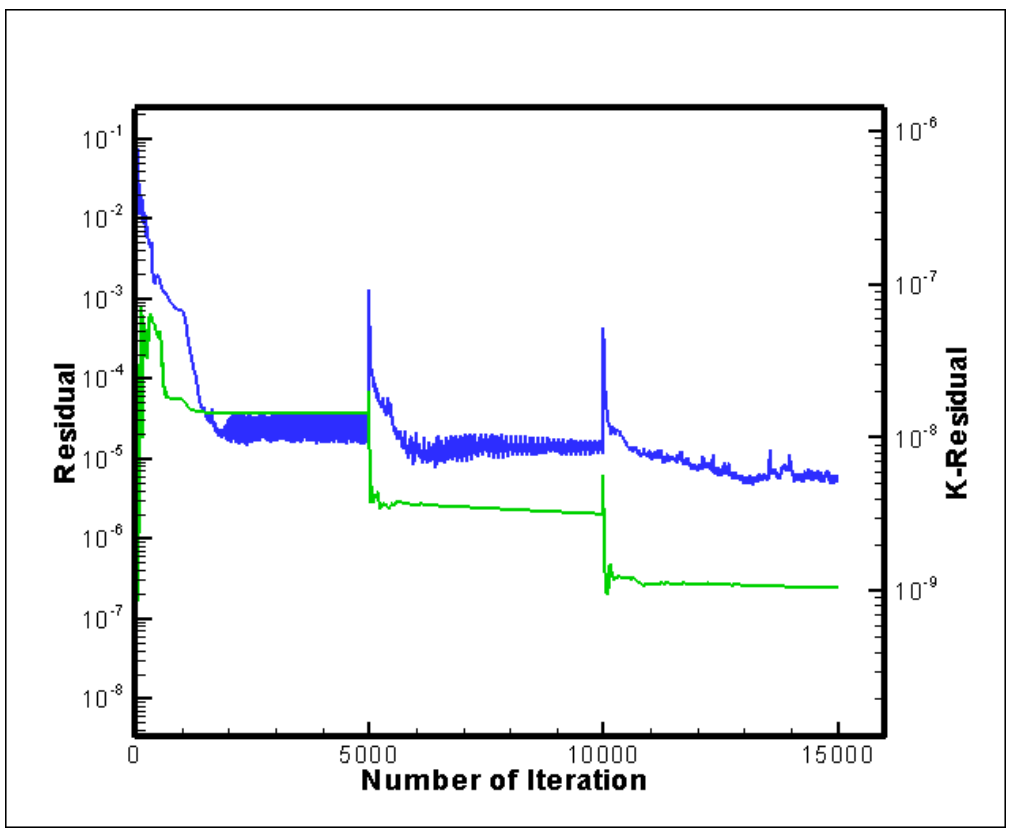

Figure 7. Convergence history for 2D nozzle flow at NPR 3.01

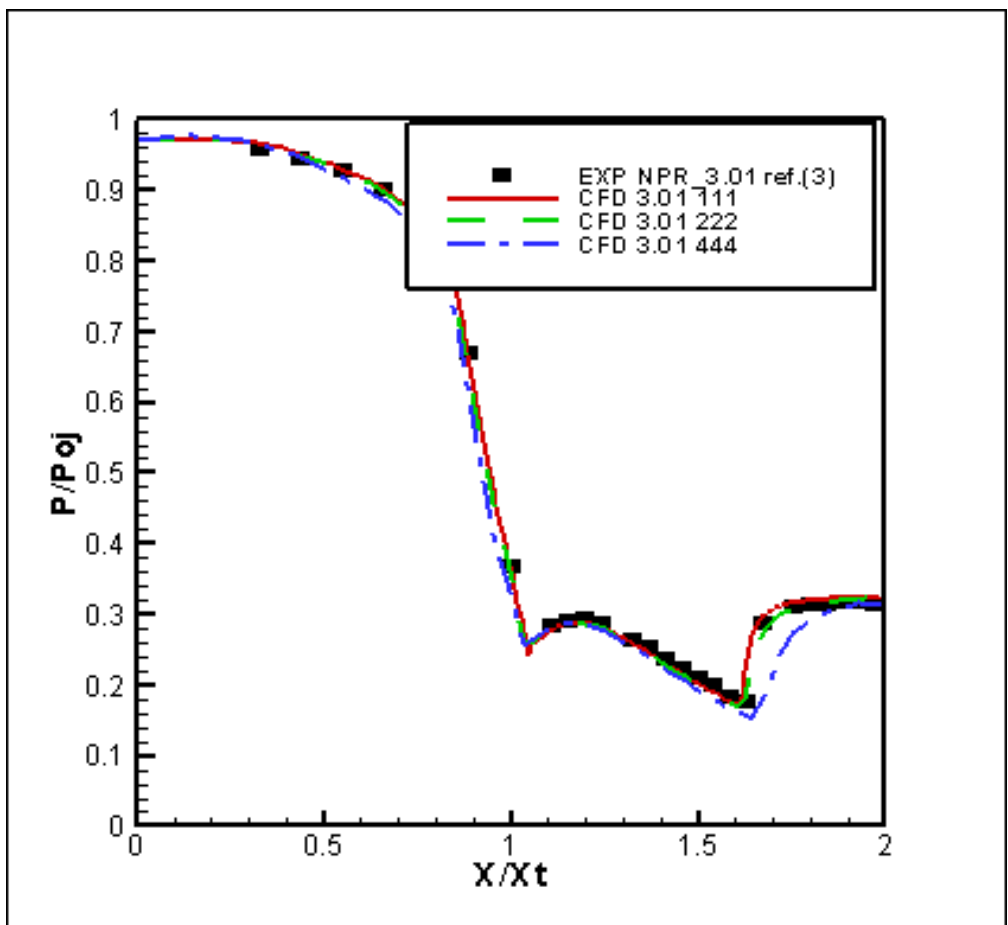

Figure 8 Comparison of computational pressure data at different grid levels for NPR $=3.0$ with experimental data of Ref. (3) 


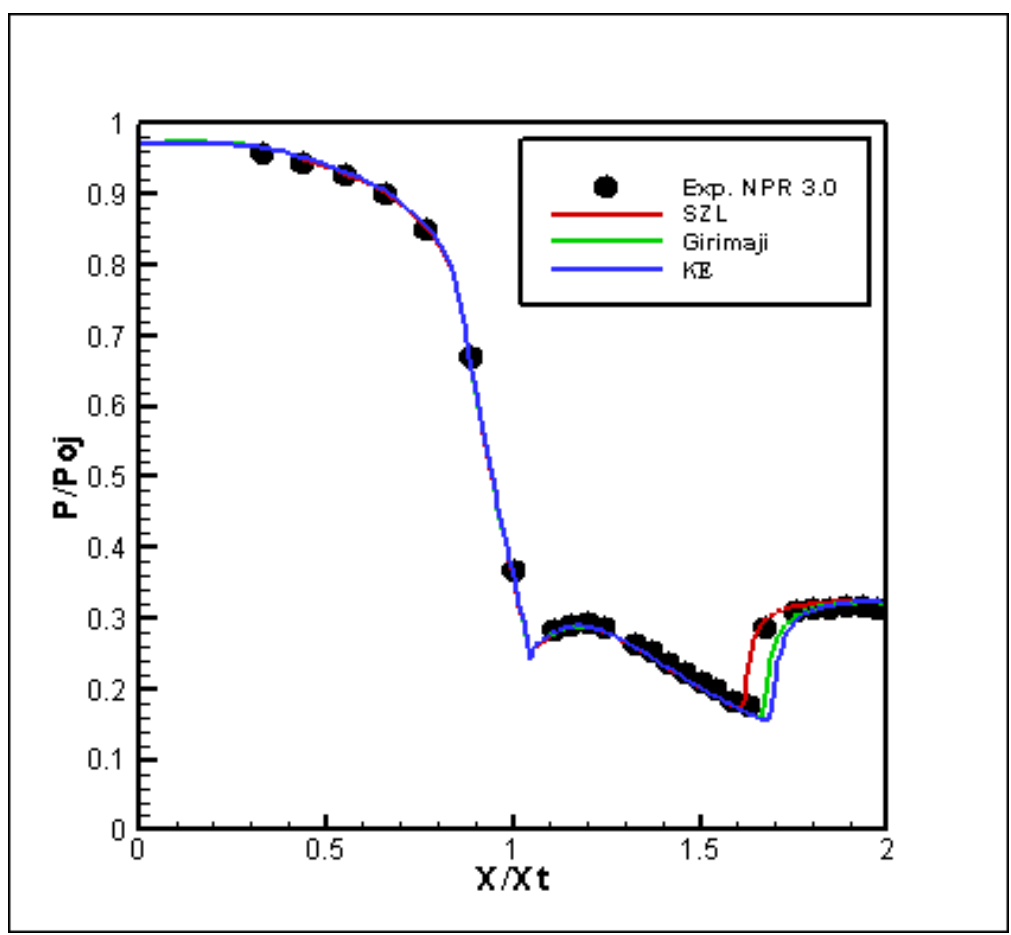

Figure 9. Comparison of computational and wall centerline experimental pressures over the entire nozzle length at $\mathrm{NPR}=3.0$

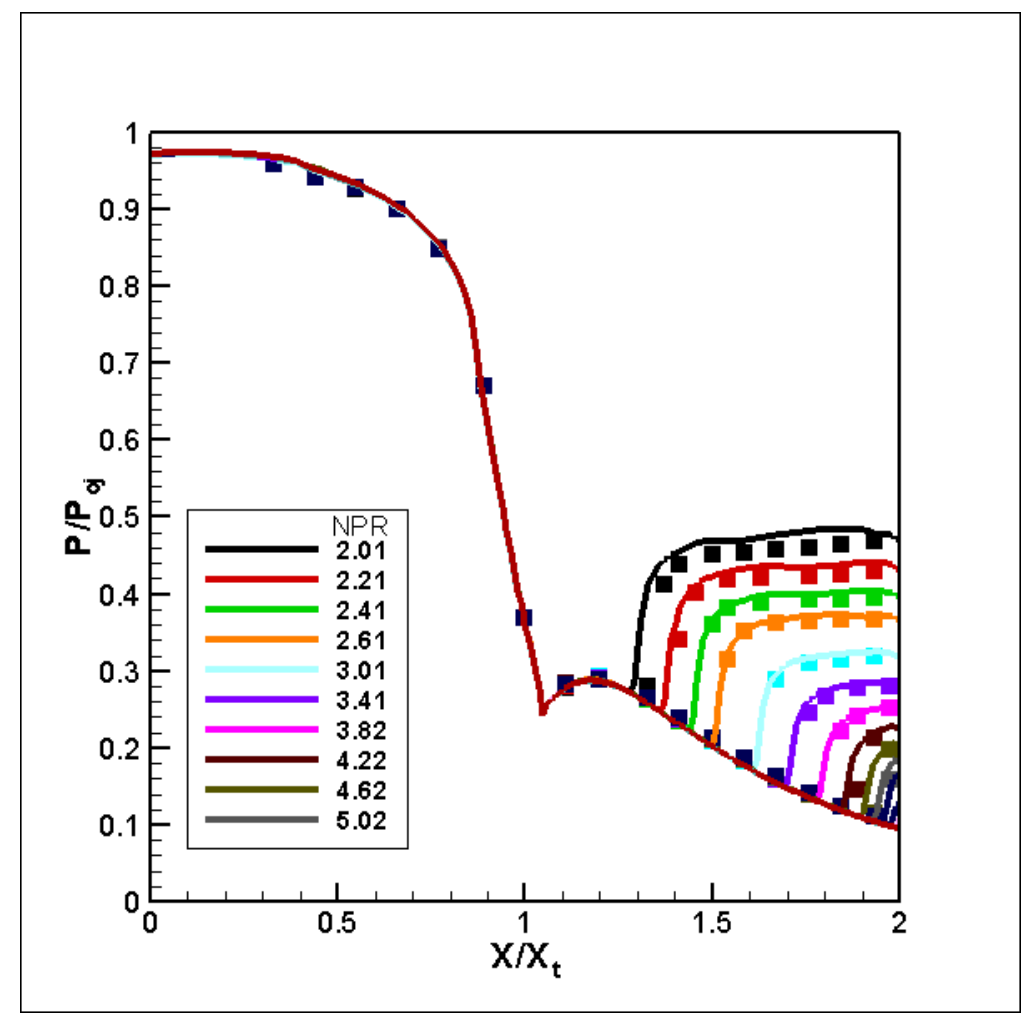

Figure 10. Comparison of computed pressure data, lines, and experimental data, symbols, of Ref (3). 


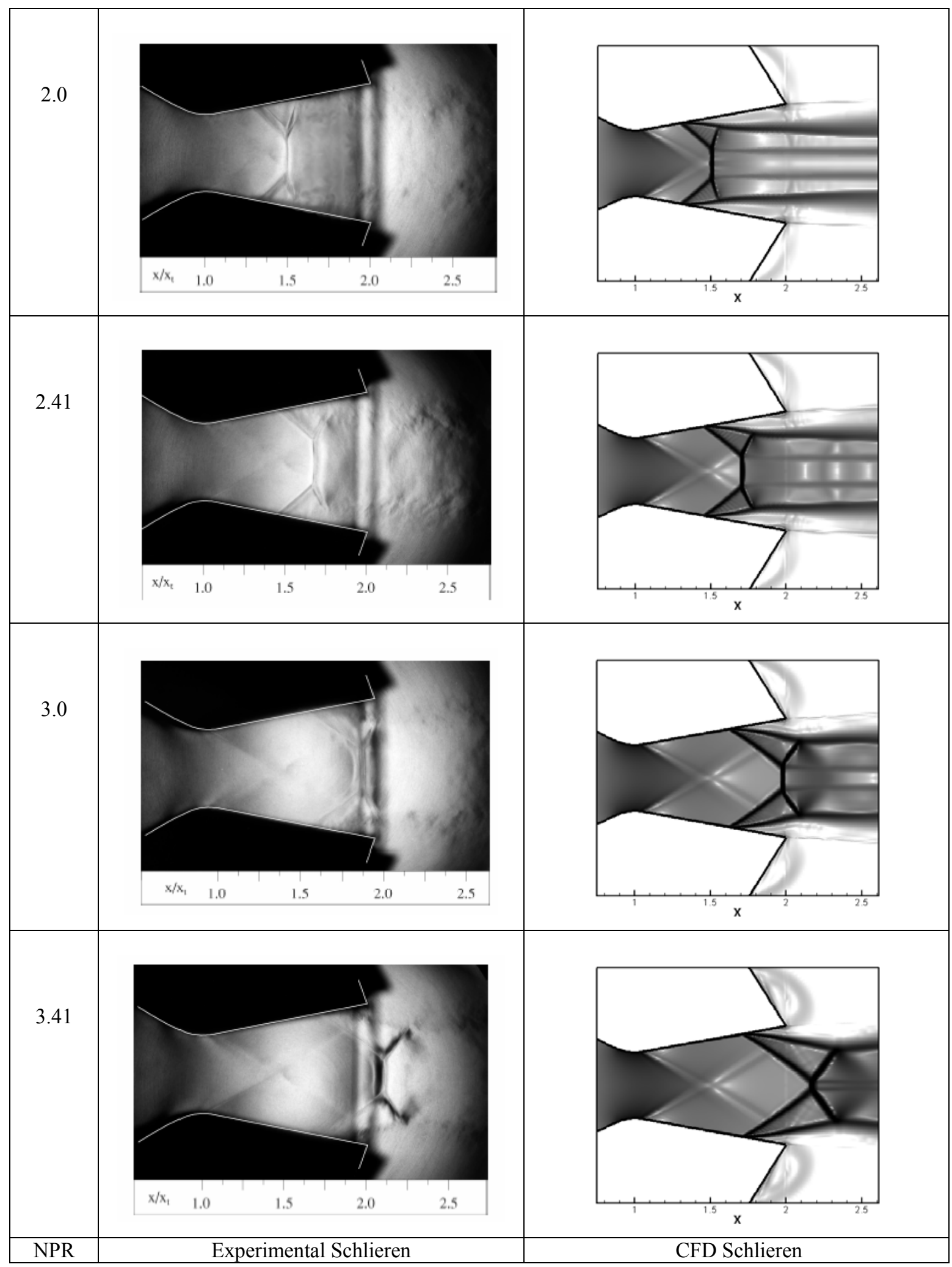

Figure 11. Comparisons of experimental and computational schlieren images for baseline nozzle configuration 


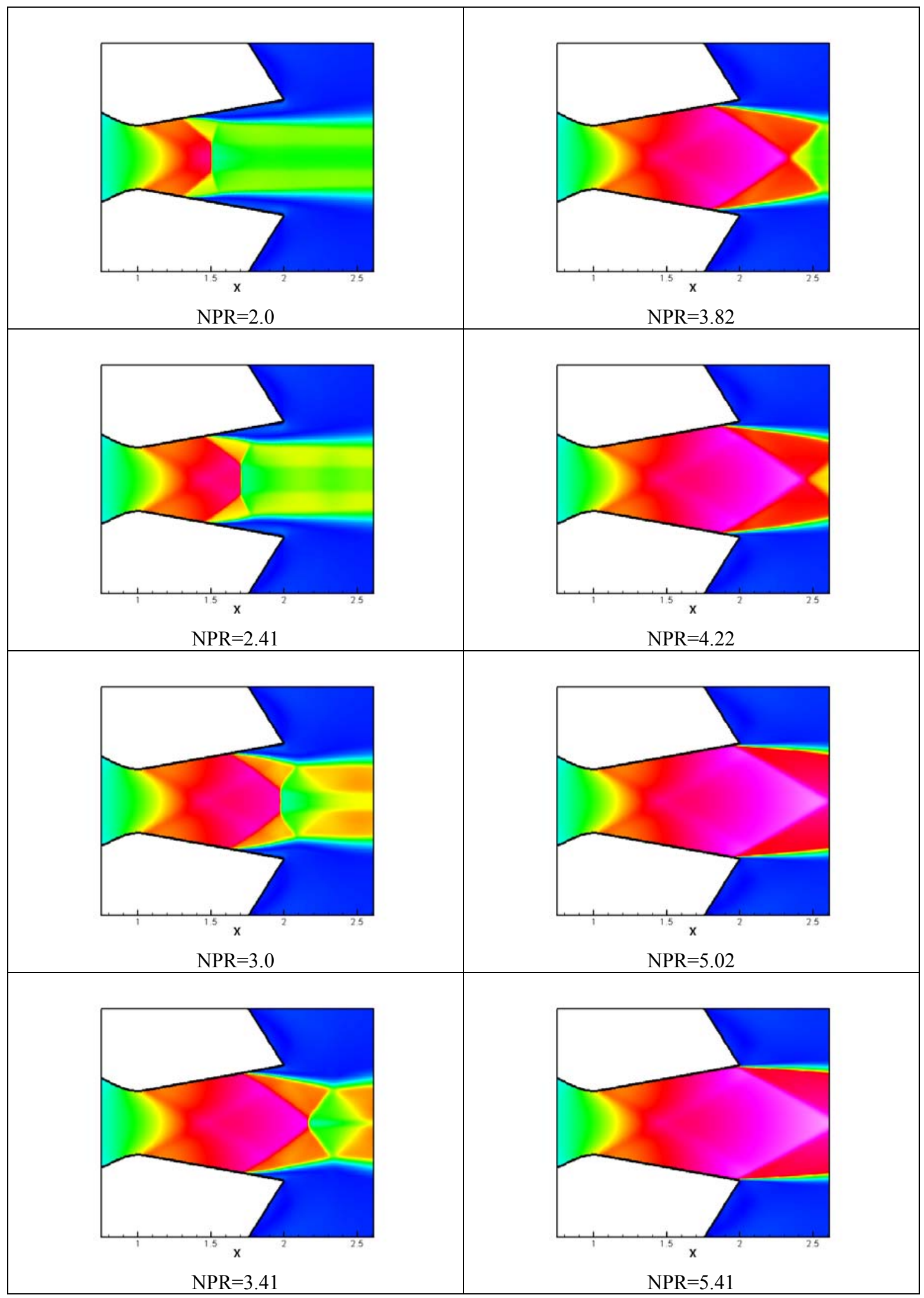

Figure 12. Mach contours for baseline configuration at various NPRs 


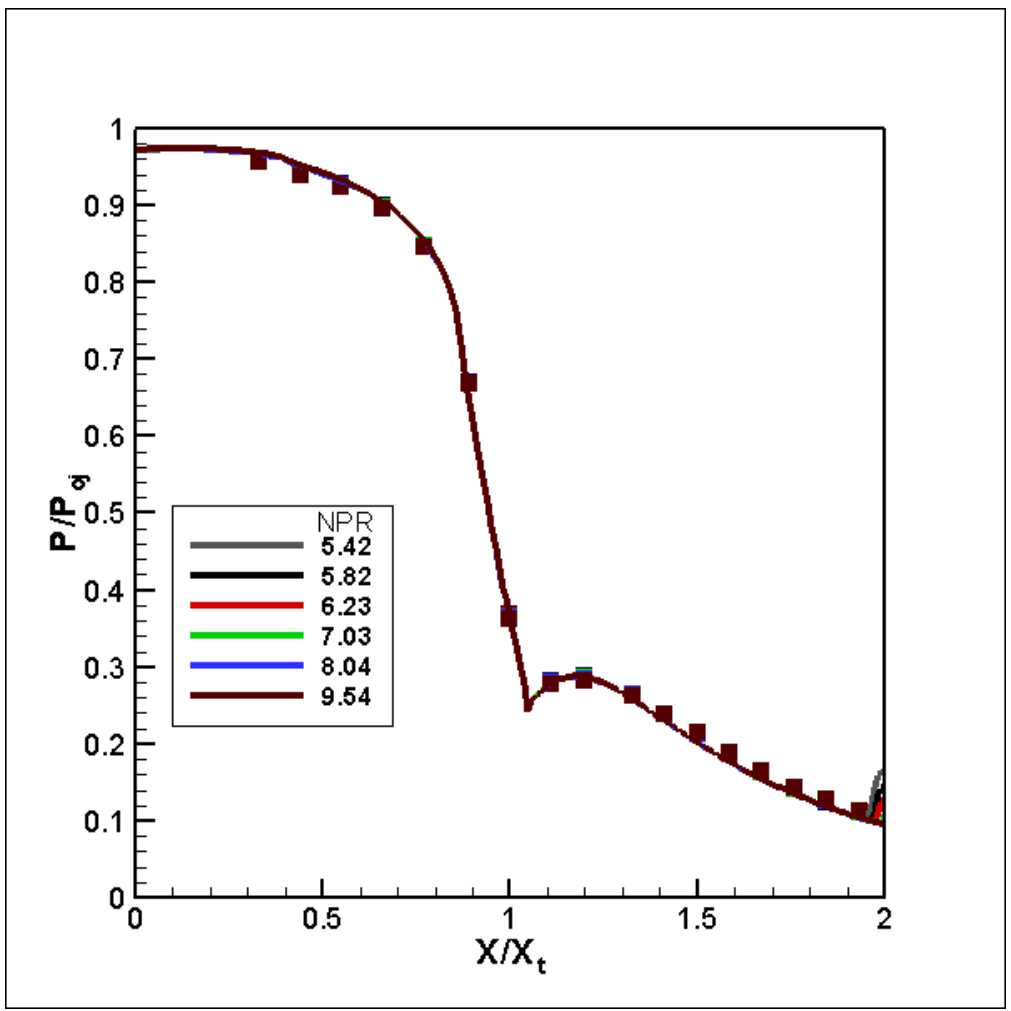

Figure 13. Comparison of computed, lines, and experimental wall centerline pressure data of Ref. (3), symbols, for NPR $\geq 5.4$ 


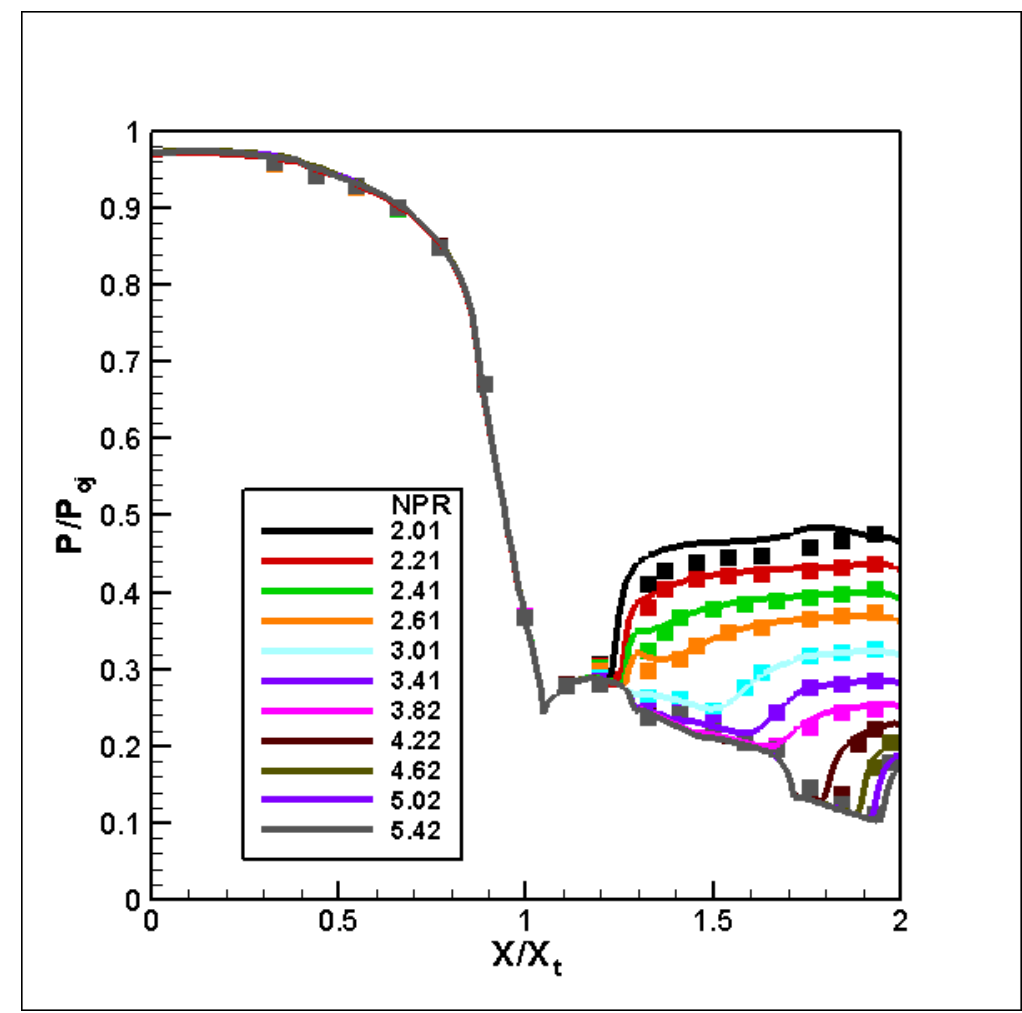

Figure 14. Comparison of computed, lines, and experimental pressure data of Ref. (3), for nozzle configuration with $10 \%$ porosity patch.

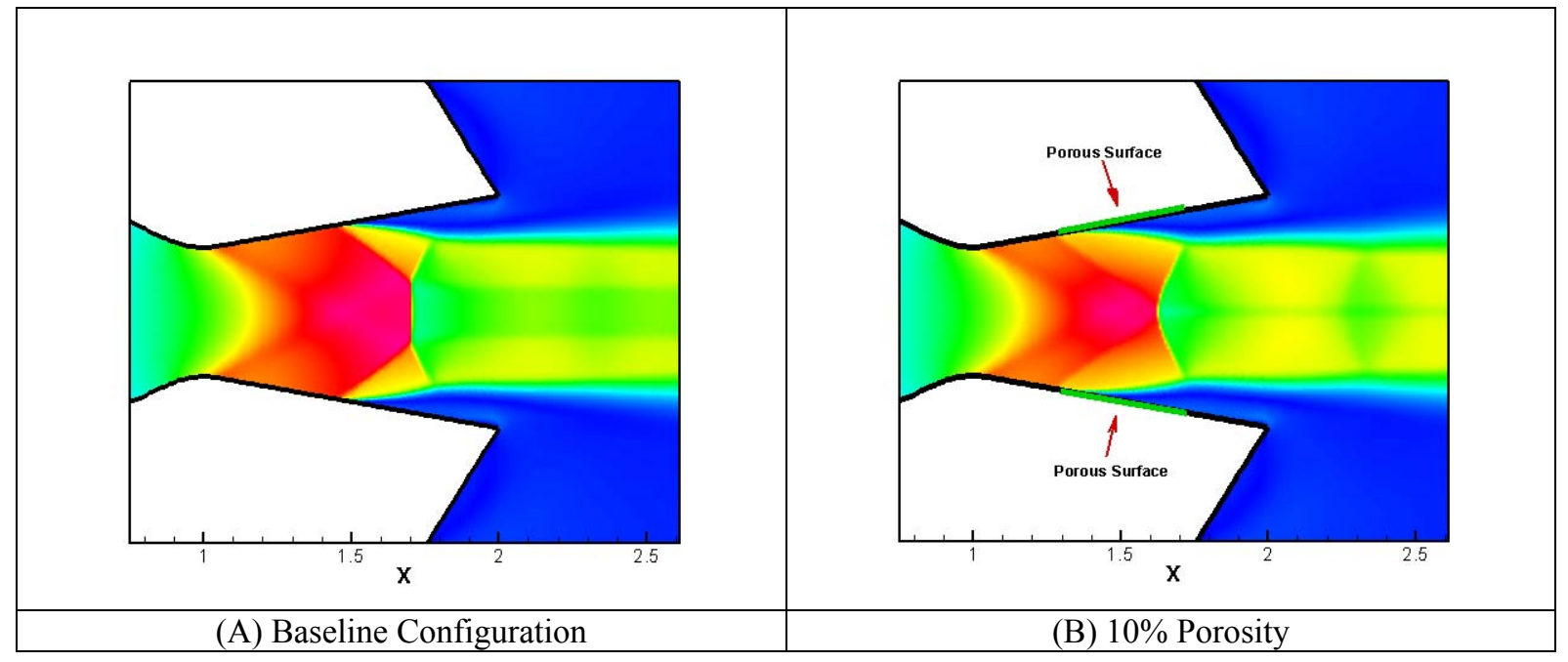

Figure 15. Contours of nozzle Mach number at NPR=2.41 


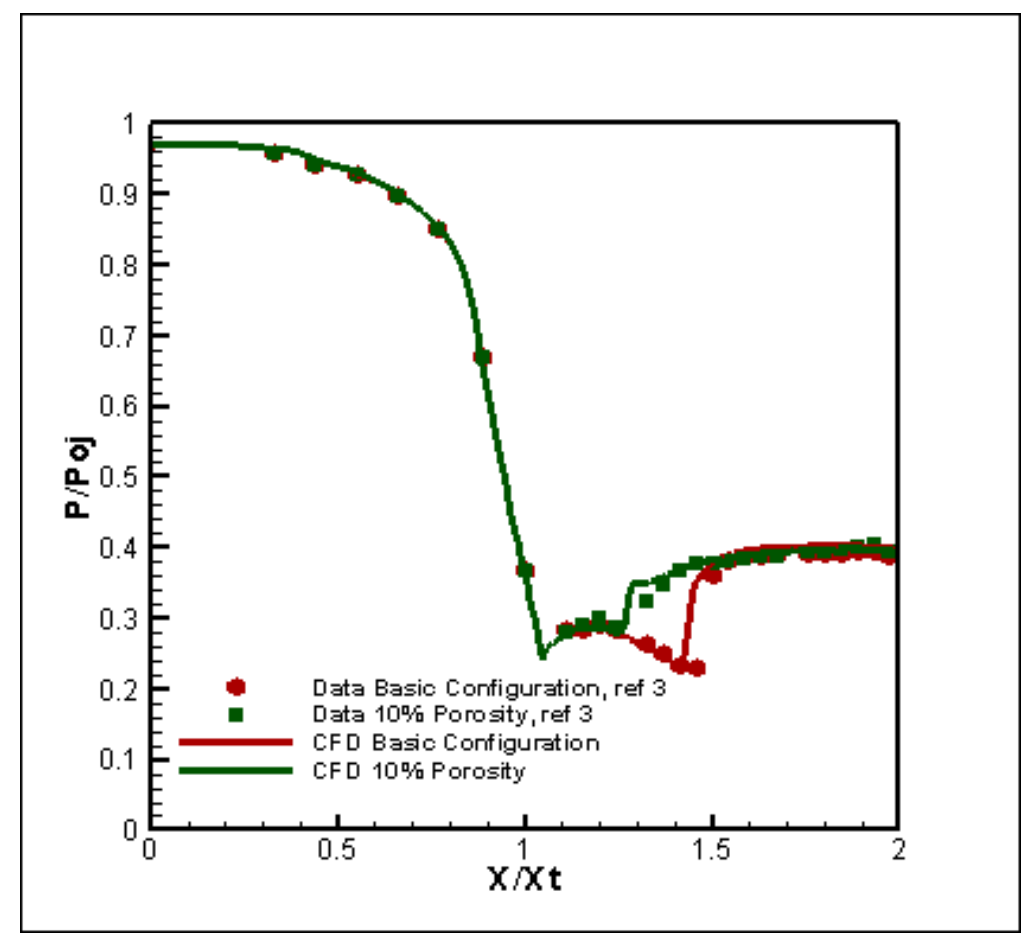

(a) $\mathrm{NPR}=\mathbf{2 . 4 1}$.

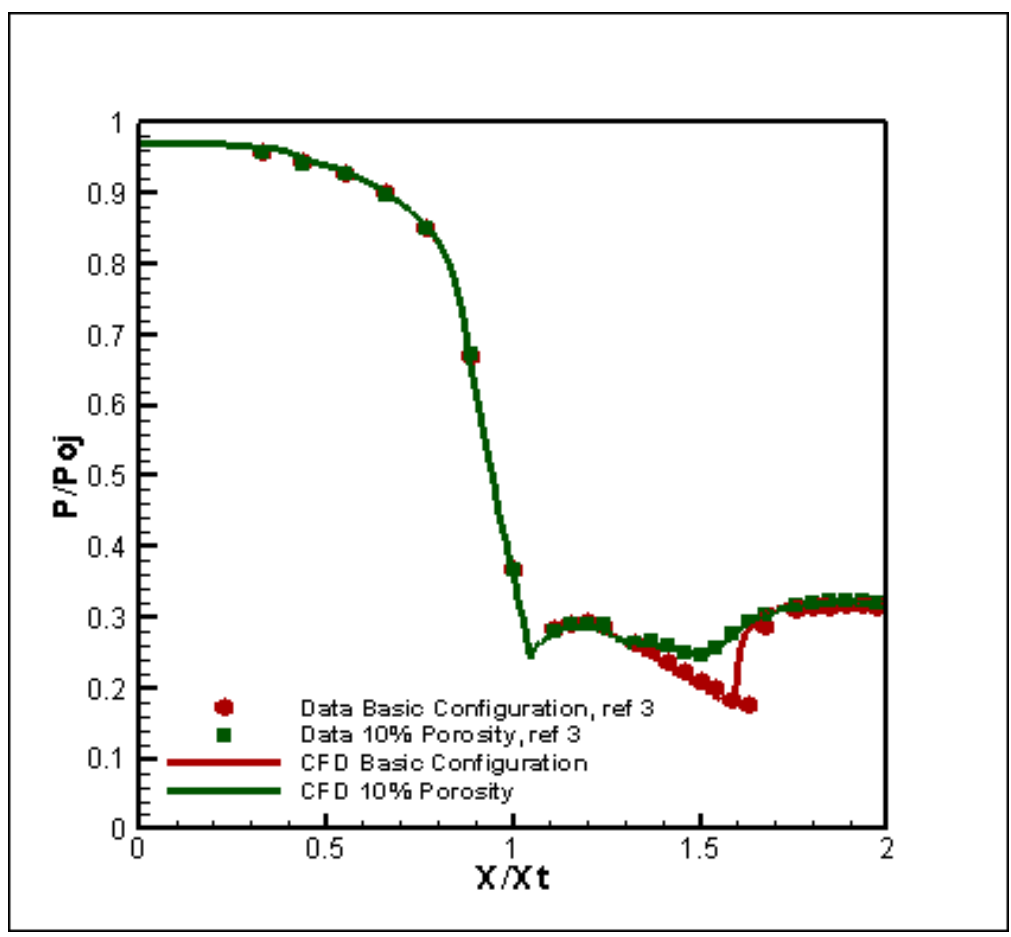

(b) $\mathrm{NPR}=3.01$.

Figure 16. Comparison of internal static pressure ratio distributions for baseline configuration and porous configuration at nominal $N P R=2.41 \& N P R=3.0$. 


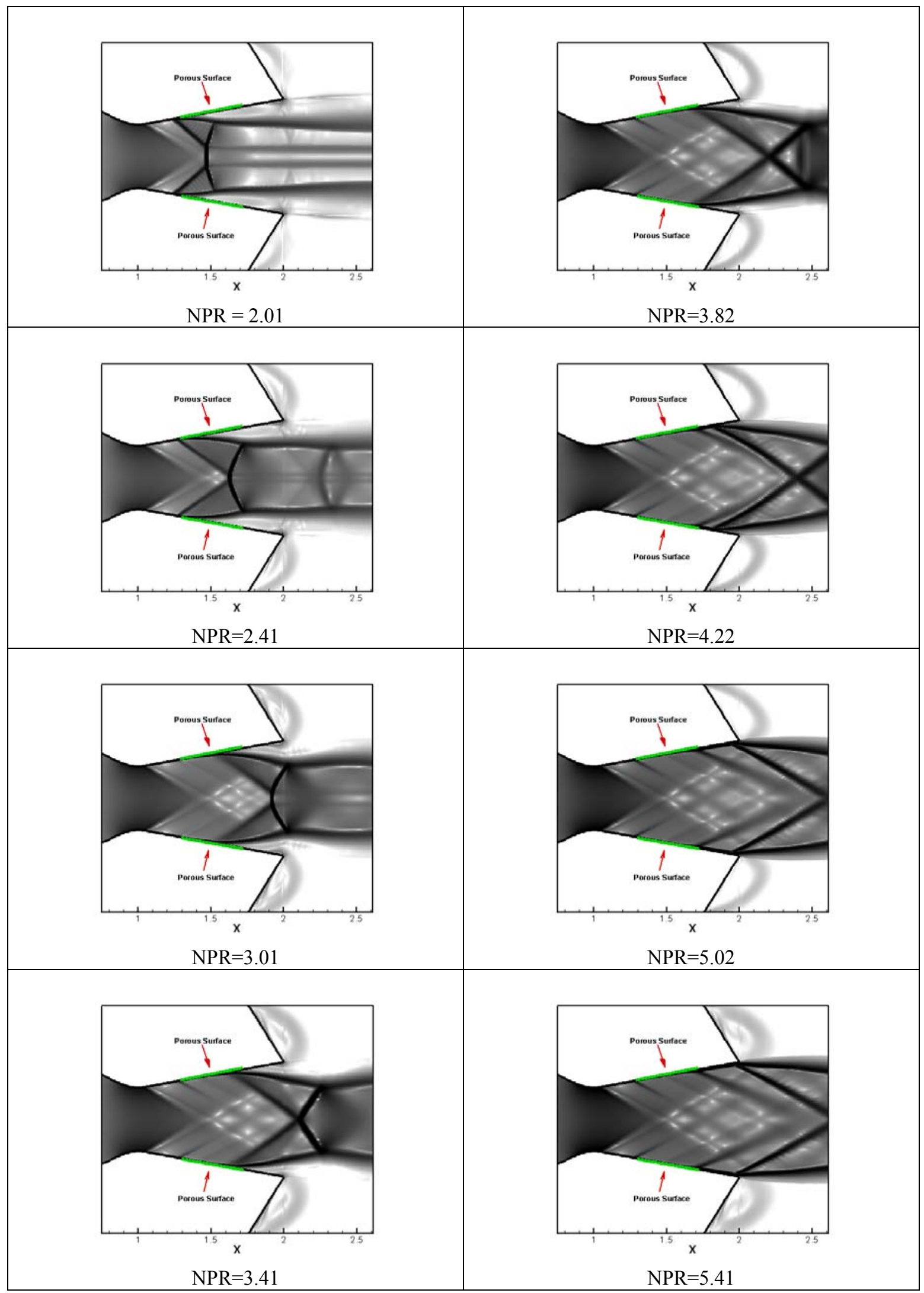

Figure 17. Computational schliern images for nozzle flow with $10 \%$ porosity ratio 

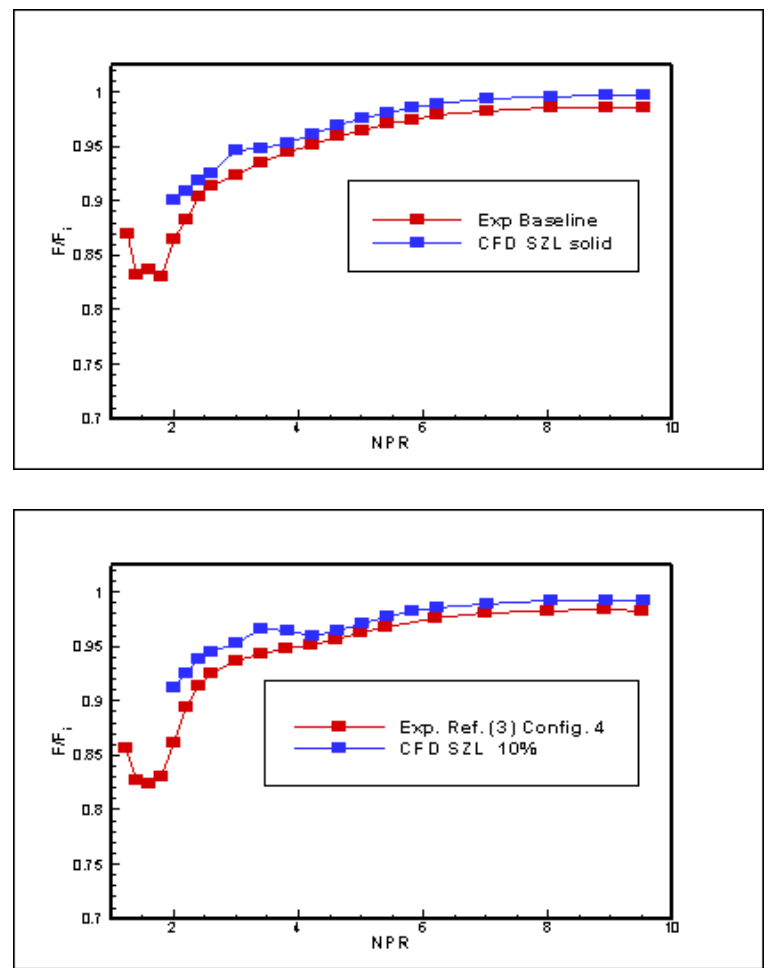

Figure 18 Comparison of nozzle thrust efficiency for baseline configuration and porous configurations and experimental data of Ref (3) 